\title{
Creating Metaphors for Tangible User Interfaces in Collaborative Urban Planning: Questions for Designers and Developers
}

\author{
Valérie Maquil, Olivier Zephir, Eric Ras \\ Public Research Centre Henri Tudor, 29, avenue John F. Kennedy, L-1855 Luxembourg, \\ Luxembourg \\ \{valerie.maquil, olivier.zephir, eric.ras\}@tudor.lu
}

\begin{abstract}
Designing tangible user interfaces (TUIs) means to deal with a complex number of issues related to the particular mixture of the physical and digital space. While a number of existing guidelines and frameworks propose issues and themes that are relevant during design, we still miss a more specific guidance on how to address such issues. This chapter analyses the difficulty of designing and developing TUIs by considering the principle of metaphors. Based on an analysis of the different types of targets of metaphors in TUIs, we identify the complexities of TUI adoption by users across physical, digital, and application domains. We propose a series of questions that support designers and developers in dealing with these complexities in the context of a TUI for collaborative planning and discussion of urban concepts. Our work is based on and illustrated through various insights collected during the development of the "ColorTable", a complex TUI for collaborative urban planning.
\end{abstract}

Keywords: Tangible user interfaces, interaction design, metaphor, design processes, urban planning

\section{Introduction}

The concept of Tangible User Interfaces (TUIs) allows us to go beyond the limitations of desktop computing and to create new applications based on interactions with a physical environment. TUIs provide the same computational capabilities as desktop computers, but are able to offer the richness and familiarity of physical actions. We believe that the strength of TUIs lies within this particular mixture of physical and digital interactions and representations. Through the physical space, we can create social effects, such as an increased collaboration (e.g., [7]), whereas the interactive representations mixing physical and digital elements offer new types of human experiences and understanding (e.g., [12])

Due to this intense combination of physical and digital interactions and representations, we are faced with a new complexity related to the design of TUIs. To deal with this complexity, researchers from diverse directions identified the need for 
general approaches to the description, the design, the implementation, and the evaluation of TUIs. In literature, we find a number of frameworks, guidelines, models, or taxonomies that all provide a certain type of guidance during design, evaluation, and description of different types of TUI systems (e.g., [1, 7, 9, 22]).

As noted by Mazalek and Van den Hoven [18], each of the frameworks provides a different perspective on tangible interaction design and supports designers and developers in a different way. We agree with Hornecker [6] that the existing frameworks tend to be theoretic and abstract and are difficult to use as step-by-step guidance in practice. Current works provide interesting lists of issues or themes that should be considered during design, whereas a more specific guidance for solving or addressing these issues throughout a design process is still missing.

This chapter approaches the tangible interaction space by drawing on theories of external and distributed cognition, i.e., by considering that cognitive processes are distributed and coordinated between internal and external elements of our environment [5]. Hence, in our work, we refer to the information and knowledge that is available in both the people's mind and and the world: We consider actions and behaviour to be caused by "a combination of internal knowledge and external information and constraints" [19]. Therefore, we take into account, at the same time what users intend to do, and what is represented through the physical and digital space of a TUI.

To be able to analyse these two types of knowledge, we focus on the different types of metaphors and metaphorical links that can be found in a tangible user interface. We believe that this approach allows us to get an understanding on the multiple dimensions of a TUI and to formulate a workflow that integrates them. Our aim is to provide practical guidance for those who need to design and develop a new TUI to support activities within a contextual and social environment. The structure and workflow presented in this chapter is a first step in identifying a sequence of the main questions to be addressed in the design activities. We invite everyone to contribute to this structure, namely to refine and complete the questions, and to provide methods and guidance for solving them.

The questions and sequence provided in this chapter are based on the multiple experiences made from the four years iterative development of the ColorTable [15, 16]. As this TUI was built to support collaborative planning and discussion of urban projects, the work primarily serves as guidance for this type of application context. In future work, we seek to generalize and adapt the workflow to a larger range of use cases.

We specifically address our work to designers and developers, since our approach requires skills from several disciplines. In our analysed context of the ColorTable, the main skills needed to advance in the design were found in design and computer science.

This chapter starts with the related concepts and works that provided us with the insights to define a series of questions: the principles of metaphors and affordances, and the complex TUI called ColorTable. We base our work on the idea that we are confronted with three types of domains (i.e., physical, digital, and application) when using or designing TUIs. Through analysing the types of targets the user needs to deal with when adopting a TUI, we define a set of issues related to user understanding of a TUI. We propose a series of questions that designers and developers need to answer 
when defining metaphors in TUIs. We finish the chapter with our conclusions and future work.

\section{Background}

\subsection{Metaphors}

The idea of metaphors has been a central and popular element in the design practice of graphical user interfaces. In our work, we use the most common and popular definition, which is based on a cognitive approach, considering a metaphor as a basic mental operation. It was formulated by Lakoff and Johnson: "A metaphor is a rhetoric figure, whose essence is understanding and experiencing one kind of thing in terms of another [14]". In 1987, Johnson provides an even more specific definition, stating that a metaphor is "a pervasive mode of understanding by which we project patterns from one domain of experience in order to structure another domain of a different kind [11]." Based on these definitions, the concept was intensively elaborated and discussed to become a key component in the design of graphical user interfaces. In TUI research, the idea was caught in several works, claiming that the physical properties of objects and space are particularly interesting for metaphorical links $[4,8,20]$.

Our work is based on these previous works on TUI metaphors and interface metaphors in general $[9,13]$, which support us in understanding how the physical, digital, and application domains are connected. Based on this understanding, we identify the design activities for creating TUI metaphors and to structure a design workflow spanning multiple domains.

\subsection{The ColorTable}

To be able to study the design of metaphors in TUIs, we take the ColorTable [15] as example. The ColorTable is a TUI enabling the collaborative discussion and debate of different ideas and concepts around urban planning projects. It is a tabletop interface presenting a collaborative planning and discussion space - users are motivated to share their ideas and visions by moving colour tokens of different shapes and colours on the table. The tokens enable users to set urban elements such as buildings, streets, pedestrian flows, or ground textures. The table view uses a physical map, which is augmented with digital information to provide a top-down view onto the project site. A vertical projection renders the scene against a background, which is produced by either a video stream, a panorama image of a view onto the site, or a see-through installation and creates a perspective mixed reality view.

The ColorTable was developed in a participatory design process of four years, and passed eight iterations, each covering the identification of the needs, the design, the implementation of interactive prototypes, and the evaluation. The prototypes had been designed and developed in a multi-disciplinary team, collaborating with experienced urban planners, and integrating material from field works. Each of the iterations was 
connected to an on-going urban planning project, providing a range of urban issues to be of interest for participants. The prototypes were evaluated in close cooperation with users, using ethnographic and observational methods within the scope of participatory workshops (Figure 1). During the different iterations, we improved the design of objects, table, and space around a) to facilitate the use for multidisciplinary groups of participants and b) to support a more and more enhanced range of application functions.

During the long-term development process of the ColorTable, we had the opportunity to experiment with different design solutions and to improve individual aspects several times. A multimodal analysis of the observational data in the multidisciplinary team, allowed us to deeply understand the characteristics of a tool, supporting groups of stakeholders to discuss urban planning decisions in a truly collaborative and participatory manner. This approach allowed us to collect and analyse a large amount of insights on the individual design activities and their importance in the development of a TUI

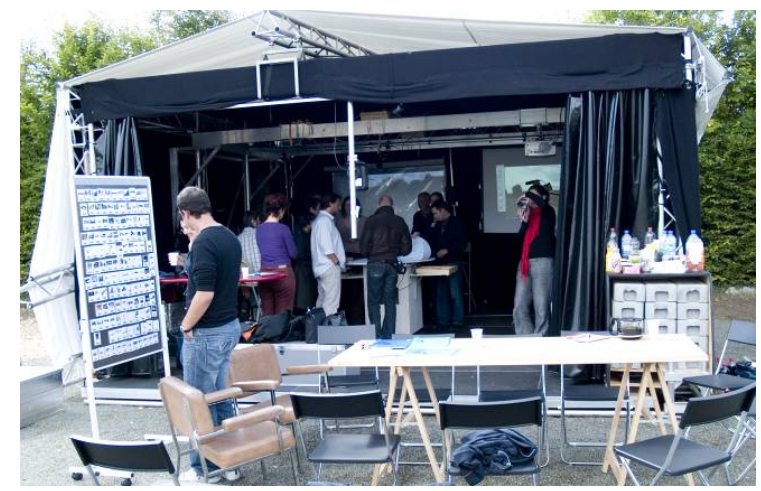

Fig. 1. Evaluating the ColorTable during participatory workshops on real sites

\section{The Complexities in a TUI}

To identify the types of complexities in a TUI, we refer to one of the most popular notion used in a metaphor definition: the source and target domain. The target is the original idea, a new or complex concept, to which the metaphor is referring to. The source is the borrowed idea, a familiar concept that helps us in understanding the target [14]. The mapping from source to target is called the metaphorical projection [13].

In the context of TUIs the source domain uses characteristics of physical objects and tangibility to refer to familiar concepts. As Fishkin [4] points out, this context is particularly appropriate for applying a metaphor-based design approach, because of the high number of physically afforded metaphors: "A designer can use the shape, the size, the colour, the weight, the smell, and the texture of the object to invoke any number of metaphorical links." 
The target domain is the unfamiliar structure in and around the TUI that needs to be understood by the user when appropriating a TUI. As described in [2], the interface can be seen as some representation that helps the user to understand the abstract operations and capabilities of the computer. This idea is in line with the work of Ullmer and Ishii $[10,21]$, comparing the TUI paradigm with the GUI paradigm. They discuss two levels, the physical and the digital level: tangible and intangible controls and representations allow users to interact with, and experience from an underlying digital information model. According to this perspective, digital information and computation is the target domain, which is presented as is it was something else that the user might already understand.

We agree with this view of seeing the interface as metaphorical representation of the operations and capabilities of the computer, but believe that in TUIs, and especially mixed reality (MR) based TUIs, we are faced with a whole series of different sources and targets that are mapped through metaphorical projections.

To better understand the different levels where complexities can be found, we analyse in more detail the way tangible interaction is structured in related literature. In his influential book "The design of everyday things" [19], Norman discusses the concept of affordances in everyday artefacts. He uses the notion of conceptual model to refer to the actions, operations, and effects of an artefact, as well as the relations in between. Affordances, constraints, and mappings allow the user to construct an own mental model of an artefact in order to understand how a system can be used. Since TUIs use everyday artefacts, the same reflexions on conceptual models can be done for TUIs: a user constructs a mental model of the TUI in order to understand how the different parts can be physically manipulated. In contrast to the digital information model of Ullmer and Ishii, the conceptual model concentrates on physical actions, and their effects, which can be considered as second type of target.

In the work of Dourish on embodied interaction [3], we can find an additional, broader view on tangible interaction. His idea behind this concept is to consider interaction as embedded in the real world. It illuminates "not just how we act on technology, but how we act through it". Artefacts of interaction can be used in many different ways and users can create different levels of meaning through their actions. He considers computation as a medium for communication; meaning is conveyed through a technology and through the practices that surround it. In other words, a transmitted meaning is something unknown and complex, which is explained through different kinds of patterns in our gestures, talk, and physical actions. This meaning created through the activities of the users can be considered as third type of target in a TUI. We name this domain as application domain.

\subsection{Case Study - The ColorTable}

To further motivate and illustrate these three different types of targets, we make use of the ColorTable. In the physical domain, the user needs to understand the physical manipulations that can, or should be done with the TUI. This includes the area where objects should be placed (i.e., the tabletop), and that they can be moved or rotated. 
The patterns used for explanation are, for example, the flat bottom side of the objects, the flat tabletop, and the directional form of the rectangles.

The second type of target concerns the computational information model, i.e., the digital domain. The user needs to build a mental model of the kind of digital objects and scenes that can be created and how they react to the different physical manipulations (Figure 2). Characteristics of the physical and digital representations support understanding how digital data is computationally represented and controlled.

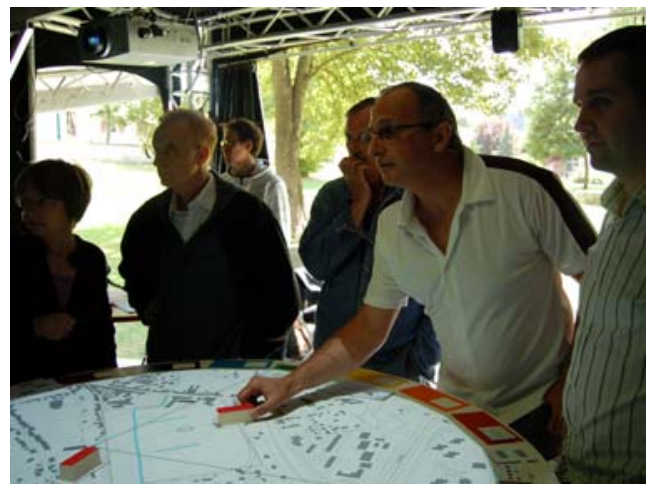

Fig. 2. Exploring and understanding how the digital space reacts to physical manipulations

Finally, a third type of target can be found in the application domain. Characteristics of the physical and the digital actions support users in conveying a meaning, i.e., expressing different kinds of properties of their urban vision. For example, they can communicate quantities, such as the position, or size of certain urban elements, but also more qualitative aspects such as the desired atmosphere, or the kind of people meeting there.

A more detailed description of these three types of targets is provided in [17].

\section{Questions for Designers and Developers}

The proposed three types of targets show the levels on which complexities in a TUI exist and need to be understood by the users. In the following, we address the three types of targets a) from the perspective of the user and b) from the perspective of the designer or developer. Based on our experiences with the ColorTable, we propose a series of questions which allow to better understand the targets of the TUI design space, and through which design activities they can be addressed. We illustrate our work through multiple observations and insights from the participatory workshops with the ColorTable. 


\subsection{Understanding the Targets of the ColorTable}

Throughout the different evaluation workshops of the ColorTable, we refined our approach of explaining the ColorTable and its different features. During the last workshops we started the sessions with a specific tutorial and developed and refined a protocol for it. The final protocol was based on four main steps, which are explained in the following.

In the first step, the starting configuration of the TUI is made, through selecting a background panorama, a paper map and 11 contents cards. The criteria for this selection are that the content gives insight into what type of urban elements the system can illustrate. However, it needs to be sufficiently neutral and not address critical issues in order to prevent users of discussing urban decisions at this point.

In the second step, the users are explained the basic principle and components of the ColorTable. This includes the table and the different tokens. The users are explained that the positions, orientations, and colours of the objects are tracked through a camera above the table, and that feedback is projected onto the map.

In the third step, the users each are provided with some of the preselected content cards. This makes them to the active user and forces them to try out the different manipulations.

In a final step, each of the features is explained individually. The list of features is made based on the different urban interventions that can be expressed through them, rather than the technical possibilities of the system. Examples for such features are: setting roads, creating zones, navigating, placing urban objects, etc. The feature is explained through a) explaining a potential use in an urban discussion, b) showing the physical move, and c) showing the digital reaction.

From this sequence, we can extract elements of how the three domains and the corresponding targets are addressed. At the beginning of the tutorial, the users are explained which physical objects are part of the system, and what physical manipulations can be done (i.e., are tracked).

When explaining the features, the users are informed on what digital objects can be created, and how they can be manipulated through which physical manipulation. The examples of digital objects are selected in a way that they reflect a typical urban planning content, i.e., a building, a bridge, or a road, in order to illustrate what types of meanings can be transmitted through the tool.

In the workshops we observed that when following this protocol, the users were able to understand the physical and the digital domain, and could proceed to selecting the objects and manipulations in order to express their own urban vision.

In contrast, on occasions where some of the tools had not been explained on the three layers, they have mostly not been used at all. A typical example of such an improper explanation is to explain the algorithm behind a digital intervention and using a "funny" example to illustrate it. Explaining that objects are visualized as pictures on invisible canvasses being placed in 3D space and illustrating this through placing a heroic figure in front of St Stevens Cathedral (the emblem of Vienna, Austria), is interesting for people which want to understand the algorithms in virtual and augmented reality. However, when users are interested in novel tools and methods for urban planning, they want to understand how the TUI can be used in this application area, and the above example is, in this case, of no help. Such an 
illustration does not show how to use the physical and digital space as metaphor for urban interventions, and requires a large mental effort of the users to imagine useful examples by themselves.

\subsection{Questions Related to Use}

The three types of targets that have been identified in the previous section imply that the design space can structured into three domains, each of them to be characterized through a type of target. When focusing on the way users approached the three targets, we can build a structure showing the unknown aspects the user faces when adopting a TUI (Figure 3).

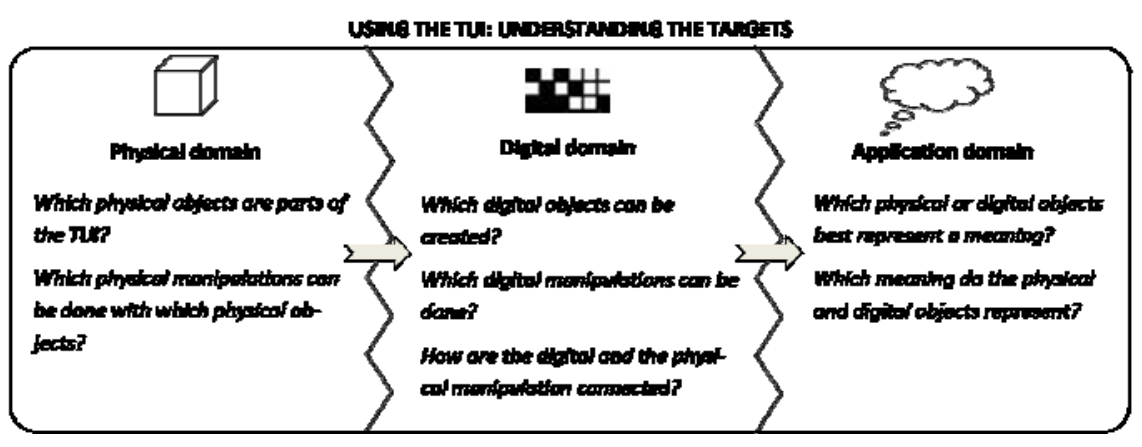

Fig. 3. Three types of targets which the user is confronted with

In the physical domain, those are about the physical objects and the respective manipulations that can be made with them. Since a TUI is always accessed in a physical space, the user first needs to find out what objects within that space are the objects of interaction (Which physical objects are parts of the TUI?). Further, s/he needs to learn how these objects can be manipulated, for example, whether they are moved, rotated, pressed, assembled, or twisted (Which physical actions can be done with which physical objects?). There may also be constraints for the manipulations, like movements that can only be done in a in a specific area, e.g., a tabletop, and other characteristics of the action, like the precision.

In the digital domain, the targets are the digital objects and the digital manipulations which can be done with them. A first unfamiliarity is what types of digital objects (i.e., shapes, lines, images, sound) can be produced with the TUI (Which digital objects can be created?). Second, the user needs to find out which actions can be done with those digital objects, i.e., whether they be moved, rotated, coloured, scaled, or distorted, and to what extend and how precisely (Which digital actions can be done with which digital objects?). Finally, s/he needs to learn which physical manipulation has which effect on which digital object (How are the physical and the digital manipulations connected?).

In the application domain, the unfamiliar aspect is the meaning which is communicated. It needs to be expressed by one user, and interpreted by the rest of the 
group. To be able to effectively use a TUI for communicating a meaning, the user needs to find out which of the hybrid interaction elements, i.e., the augmented physical objects and manipulations, show the important aspects that characterize the meaning a user wants to convey (Which physical and/or digital objects best represent a meaning?). The rest of the group needs to interpret this representation and understand which characteristics of the shapes, images, sounds, and tokens, as well as the related manipulations, provide which information about the meaning which is communicated (Which meaning do the physical and digital objects represent?).

Guided through the tutorial sessions in our participatory workshops, the users approached the targets in the aforementioned order. They first tried to understand the physical objects and the manipulations that can be done with them. Then they sought to understand how the digital objects and manipulations can be controlled, and finally they were ready to use the tool as support to communicate about concepts and their properties (i.e., meaning) in the application domain.

\subsection{Questions Related to Design}

Based on the above specification on the different levels of understanding of the user, we are able to define design questions which directly address these targets (Figure 4).

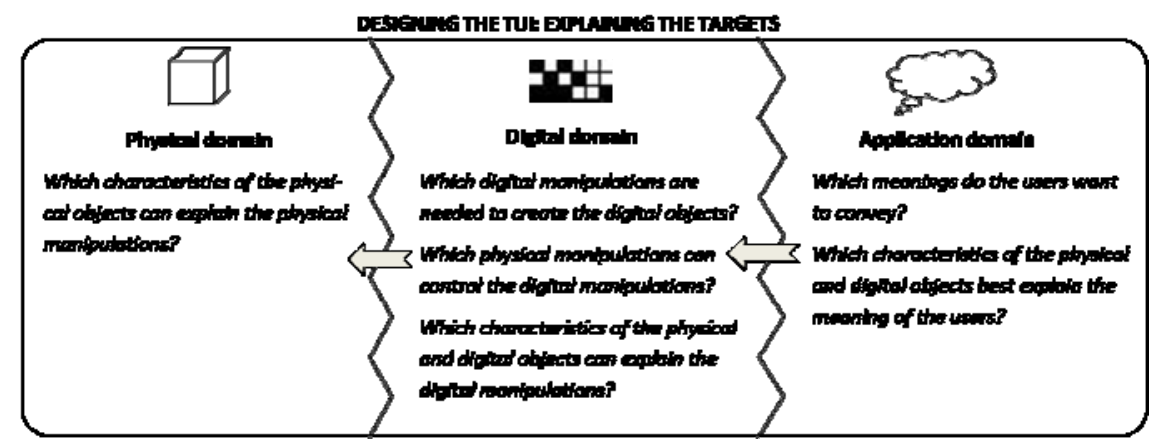

Fig. 4. Designing the TUI based on the tree types of targets the user is confronted with

To explain the application domain, we need to understand and specify the meanings users can convey and define metaphors for expressing them with the TUI. As a first step, we need to elaborate the topics the users want to address and discuss, as well as how precisely they want to discuss them (Which meanings do the users want to convey?) Further, a decision needs to be taken on how each of the topics and related qualities of discussion is represented through physical and digital objects and manipulations, i.e., what types of images, shapes, sound, physical objects refer to the important characteristics of the conveyed meaning (Which characteristics of the physical and digital objects best explain the meanings?)

To explain the digital domain, we need to elaborate the digital possibilities and the corresponding metaphorical links to the physical domain. In this step, we need to find solutions for creating digital objects showing the qualities elaborated in the previous 
domain. So we need to determine the digital actions, e.g., translations, rotations, scaling procedures, distortions that are required for creating the desired digital effect and appearance (Which digital manipulations are needed to create the digital objects?). Further, we need to investigate which physical manipulations can be technically sensed, through which type of physical object this manipulation can be realized, and under which circumstances the physical manipulation can be linked to the variables of the digital manipulation (Which physical manipulations can control the digital manipulations?). Lastly, we need to think about the properties of the physical and digital objects that show the users the possibilities for digital manipulations (Which characteristics of the physical and digital objects can explain the digital manipulations?).

Finally, in the physical domain, we have to design the physical properties to explain the physical actions. We need to elaborate which properties of the physical objects show the users the possibilities for physical manipulations (Which characteristics of the physical objects can explain the physical manipulations?).

When focusing on the levels of understanding of the user, it suggests itself to approach the design space starting from the application domain, then working towards the digital and the physical domain. However, in a user-centred design perspective, several iterations and simultaneous adaptations are needed. This means that the designer also needs to redefine the outcome of each step several times and adjust design decisions based on previous evaluations and user feedback. This will, for instance, allow him to adjust decisions of the application domain based on issues of the physical domain.

\subsection{Case Study - The ColorTable}

Although this structure was not used during the development of the ColorTable, we can show that the proposed questions have all been addressed at some moment during the different iterations. In the following, we describe the issues around each of the questions, as well as our approach for solving them.

Which meanings do the users want to convey? This question was mainly addressed by the urban specialists in the team. These selected an ongoing urban planning project and suggested a set of 'urban themes' they considered as particularly relevant. They illustrated these themes by providing a number of visual examples, such as scale, temporality, borders and layers, fuzziness, ambience and mobility. Using participatory design techniques (e.g., cultural probes, narrative interviews), these issues have been detailed with user's own vision of the future site. Different scenarios of statements have then been elaborated, such as 'work with issues of connectivity', 'discuss allocation of spaces for different uses', or 'activities and ambiances'.

Which characteristics of the physical and digital objects best explain the meanings conveyed by the users? Based on the elaborated scenarios, we created and selected ways to represent the statements with digital and physical objects. This was mainly done through intense collaboration with an artist and wide exploration of different visual and technical possibilities. Outcomes of this step were, for instance, that urban elements need to be represented through photos, as these better visualize the specific details of an ambiance compared to 3D models (Figure 5). The photos need to be placed on any position in order to link the ambiance with a specific location. User 
feedback during the workshops allowed us to refine the characteristics of physical and digital objects. For instance, users were repeatedly mentioning that objects 'are not on the ground', or 'are floating' (Figure 6). We therefore identified the necessity of positioning any type of objects in a way that they are perceived to be on the ground. Further, users were mentioning the need for smaller physical objects.

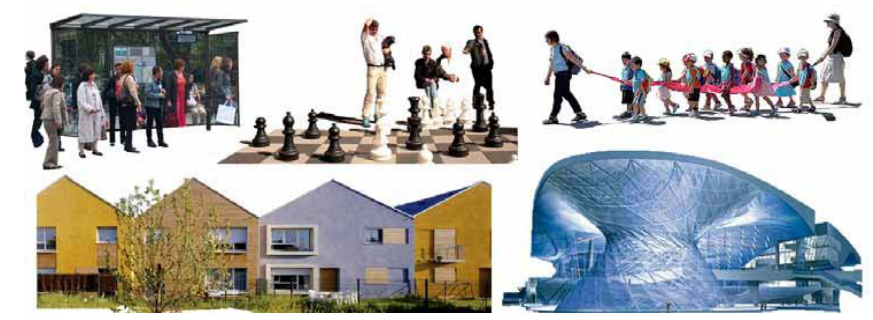

Fig. 5. Representing the specific details of an ambiance through photos

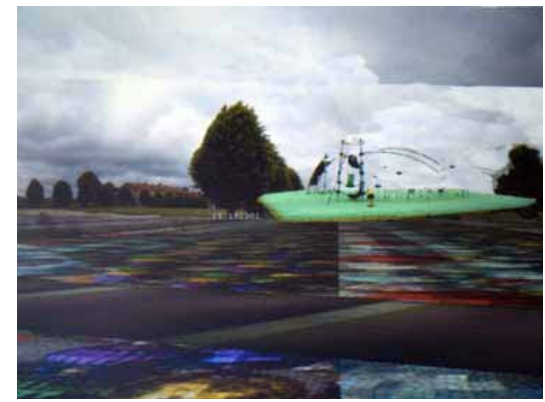

Fig. 6. A playground which is not perfectly adjusted to the background is perceived to be 'floating'

Which digital manipulations are needed to create the digital objects? In this step we defined the digital manipulations that can create the visual effects elaborated in the previous design step. This was mainly done through exploring technical possibilities, implementing them, and evaluating how they are perceived by the users. Outcome of this step was, for example, the requirement for $3 \mathrm{D}$ positioning and scaling of each object. To create the impression that a certain photo has been placed on a specific position on the ground in the panorama, the object needs to be lowered depending on the perspective in its own photo, correctly scaled, and be occluded through 'real' objects in the panorama.

Which physical manipulations can control the digital manipulations? In this step we analysed which physical manipulations can be sensed and connected to the digital manipulation. Possibilities for physical manipulations depend on the choice of the sensing technology and are created through combining a technology with different types of physical objects. In the case of the ColorTable, this step was done in an intense collaboration between designers and computer scientists, by exploring technical and physical possibilities and doing creative combinations. Outcome of this step was, for instance, the manipulation of tokens of different colours and shapes to 
control positions, and the placement of small cards on dedicated colour areas to control other parameters (Figure 7 right).

Which characteristics of the physical and digital objects can explain the digital manipulations? Knowing the possibilities for physical manipulations and the requirements for digital manipulations, we can make links between the two. When defining these links, we need to elaborate how the digital manipulations can be explained to the user by well selecting physical and digital objects. This step heavily relied on the feedback and observation of users provided in the participatory workshops. A design of metaphorical links was implemented and then iteratively adapted based on the results of the workshops. In the example of placing photos, the positioning on the XY plane (corresponding to the floor) was controlled through positioning the colour tokens on the tabletop (Figure 7 left). Since this imitates the behaviour of objects in the real world, the link is easy to understand. The manipulation of size and offset to the ground was decided to be controlled via command cards placed on RFID readers (Figure 7 right). However, this is more difficult to understand since the physical manipulation does not refer to any familiar metaphorical source. We therefore explained this manipulation digitally using textual information popping up and indicating size and offset in meters. Better metaphors for modifying the size of objects could, for instance, be based on stacking or rotating mechanisms on the objects themselves.

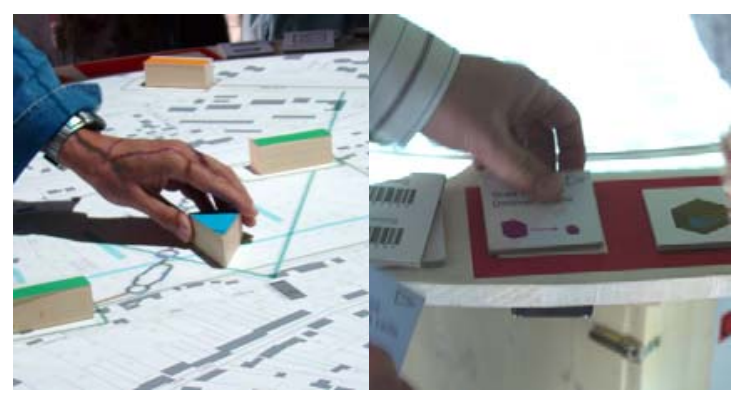

Fig. 7. Moving physical objects (left) to control positions and placing a small card on a color area (right) to increase the size

Which characteristics of the physical objects can explain the physical manipulations? In this step, we thought about the design of the objects themselves and how they can support the user in manipulating them correctly. This was mainly done through collaboration with an industrial designer and exploration of physical possibilities as well as their alignment to requirements developed in previous steps. Outcomes were, for example, a flat bottom side that motivates the user in placing the objects on the table. A certain height allows her/him to grasp them from the side and avoid occlusion on the camera image. A certain weight infers that they are comfortable to hold in the hand while thinking.

When considering the design and development of the ColorTable, we can see the importance of each of the proposed questions and how they affect design decisions for the related domains. Although a basic approach was to define digital and physical manipulations after having elaborated an understanding of the application domain, the 
case study showed that this sequence was not followed rigidly. For instance, as mentioned before, outcomes of the 'digital' and 'physical' step have influenced decisions taken related to the application domain. The physical objects needed to have a certain size in order to be detected by the colour vision framework. This means that on a large urban site, the objects physically held a place on the map that corresponds to a large building. Because of this issue we decided that it makes no sense for using 'small' urban elements, like a tree or a bench, when the urban site is rather large.

The common strategy of the design process of the ColorTable was to start with a loose definition of each of the domains, and then specify each of the domains of the design space in more and more detail. The first prototype built upon the idea of a tabletop using physical objects and a mixed reality view to represent urban planning elements. Based on this concept, the TUI has been more and more refined in order to respond to the requirements and outcomes related to the different questions of the three domains.

\section{Conclusions and Future Work}

This chapter proposes a range of questions which support designers and developers in designing TUIs while considering both the internal knowledge in the users' mind, and the external knowledge represented on the tabletop. Our work is based on the different insights collected during the iterative and participatory design process of the ColorTable. The principle of metaphors and the elaborated three types of targets allowed us to consider aspects of the physical, the digital, and the application domain in TUIs.

In our discussion we provided examples on how and why the different questions had been addressed during the design and development of the ColorTable. The approach shows that each of the related steps features an important complexity which can be managed using skills of multiple disciplines.

Structuring the design space in three domains allowed us to identify a range of questions to be integrated in the design process of a TUI, addressing issues of its usage as a tool within a broader context. In particular, the provided structure highlights which kinds of associations between components of the physical, digital, and application domain need to be made explicit to the user in order to ensure successful design.

Our work is a first step towards a more specific workflow of design activities in TUIs. It provides a range of issues that need to be considered during design, and points out how the different decisions are interconnected. However, there is still a lot to do. On one hand, the questions need to be completed and refined through a high number of case studies. Further, existing guidelines and methods, which support finding a solution to these questions, need to be aligned with the structure. There is also the need for new guidelines and methods to fill currently existing gaps. While we provide first hints on a kind of sequence for addressing the questions, this workflow needs to be elaborated further in order to create a more effective and efficient design process.

In our future work, we will test and refine the questions in two different case studies dealing with collaborative service design activities using tabletops. Our aim is 
to complete and refine the questions and to develop a design support for this particular domain. We believe that through our metaphor-based approach to TUIs, linking multiple domains, we will be able to define a design workflow, which allows us to more effectively and efficiently design new TUIs.

Acknowledgments. The majority of the research underlying this chapter was conducted by the first author as Ph.D work within Vienna University of Technology. It was partially funded by the European Union Integrated Project IPCity under contract no. FP6-2004-IST-4-27571. We would like to thank all researchers who contributed to the work of this chapter, in particular the team of Vienna UT: Lisa

Ehrenstrasser, Stephan Gamohn, Michal Idziorek, Ina Wagner, and Mira Wagner.

\section{References}

1. Antle, A.N.: The CTI Framework : Informing the Design of Tangible Systems for Children. Proceedings of the 1st international conference on Tangible and embedded Interaction. pp. 15-17 ACM (2007).

2. Blackwell, A.F.: The reification of metaphor as a design tool. ACM Transactions on Computer-Human Interaction (TOCHI). 13, 4, 490-530 (2006).

3. Dourish, P.: Where the action is: the foundations of embodied interaction. The MIT Press (2004).

4. Fishkin, K.: A taxonomy for and analysis of tangible interfaces. Personal and Ubiquitous Computing. 8, 5, 347-358 (2004).

5. Hollan, J., Hutchins, E., Kirsh, D.: Distributed cognition: toward a new foundation for human-computer interaction research. ACM Transactions on Computer-Human Interaction. 7, 2, 174-196 (2000).

6. Hornecker, E.: Creative idea exploration within the structure of a guiding framework: the card brainstorming game. Proceedings of the fourth international conference on Tangible, embedded, and embodied interaction. pp. 101-108 ACM (2010).

7. Hornecker, E., Buur, J.: Getting a grip on tangible interaction: a framework on physical space and social interaction. Proceedings of the SIGCHI conference on Human Factors in computing systems. pp. 437-446 ACM (2006).

8. Hurtienne, J., Israel, J.H.: Image schemas and their metaphorical extensions: intuitive patterns for tangible interaction. Proceedings of the 1st international conference on Tangible and embedded interaction. pp. 127-134 ACM (2007).

9. Hurtienne, J., Weber, K., Blessing, L.: Prior experience and intuitive use: image schemas in user centred design. Designing inclusive futures. (2008). 
10. Ishii, H.: Tangible bits: beyond pixels. Proceedings of the 2nd international conference on Tangible and Embedded Interaction. (2008).

11. Johnson, M.: The Body in the Mind. The University of Chicago Press (1987).

12. Klemmer, S.R., Hartmann, B., Takayama, L.: How bodies matter: five themes for interaction design. Proceedings of the 6th conference on Designing Interactive systems. pp. 140-149 ACM (2006).

13. Kuhn, W., Frank, A.U.: A formalization of metaphors and image-schemas in user interfaces. Cognitive and linguistic aspects of geographic space. 63, 419-434 (1991).

14. Lakoff, G., Johnson, M.: Metaphors we live by. Chicago London (1980).

15. Maquil, V.: The ColorTable : an interdisciplinary design process. Vienna University of Technology (2010).

16. Maquil, V., Psik, T., Wagner, I.: The ColorTable: a design story. Proceedings of the 2nd international conference on Tangible and Embedded Interaction. pp. 97-104, Bonn, Germany (2008).

17. Maquil, V., Ras, E., Zephir, O.: Understanding the Characteristics of Metaphors in Tangible User Interfaces. Mensch \& Computer 2011. 47.

18. Mazalek, A., van den Hoven, E.: Framing tangible interaction frameworks. Artificial Intelligence for Engineering Design, Analysis and Manufacturing. 23, 03, 225 (2009).

19. Norman, D.A.: The Design of Everyday Things. Basic Books (2002).

20. Svanaes, D., Verplank, W.: In search of metaphors for tangible user intefaces. Proceedings of DARE 2000 on Designing augmented reality environments. pp. 121129 ACM (2000).

21. Ullmer, B.: Emerging frameworks for tangible user interfaces. IBM systems journal. 39, 915-931 (2000).

22. Zuckerman, O., Arida, S.: Extending tangible interfaces for education: digital montessori-inspired manipulatives. Proceedings of the SIGCHI conference on Human factors in computing systems. (2005). 